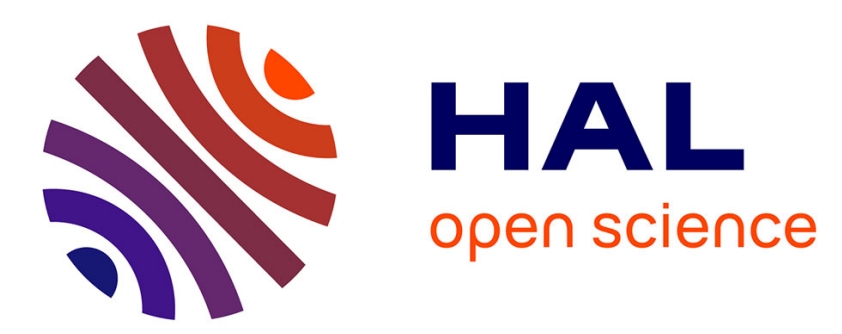

\title{
Determination of the thermophysical properties of evolutive porous media: application to Civil Engineering materials
}

Philippe Poullain, Pierre Mounanga, Guy Bastian, Roger Coue

\section{- To cite this version:}

Philippe Poullain, Pierre Mounanga, Guy Bastian, Roger Coue. Determination of the thermophysical properties of evolutive porous media: application to Civil Engineering materials. European Physical Journal: Applied Physics, 2006, 33, pp.35-49. 10.1051/epjap:2005095 . hal-01006734

\section{HAL Id: hal-01006734 \\ https://hal.science/hal-01006734}

Submitted on 3 Feb 2018

HAL is a multi-disciplinary open access archive for the deposit and dissemination of scientific research documents, whether they are published or not. The documents may come from teaching and research institutions in France or abroad, or from public or private research centers.
L'archive ouverte pluridisciplinaire HAL, est destinée au dépôt et à la diffusion de documents scientifiques de niveau recherche, publiés ou non, émanant des établissements d'enseignement et de recherche français ou étrangers, des laboratoires publics ou privés. 


\title{
Determination of the thermophysical properties of evolutive porous media: application to Civil Engineering materials
}

\author{
P. Poullain, P. Mounanga ${ }^{a}$, G. Bastian, and R. Coué \\ GeM, UMR CNRS 6183, Institut de Recherche en Génie Civil et Mécanique, IUT de Saint-Nazaire, Département Génie Civil, \\ BP 420, 44606 Saint-Nazaire Cedex, France
}

\begin{abstract}
The purpose of this paper is to describe the development and the use of two measurement techniques especially adapted to the rapid determination of the thermophysical properties of evolutive porous media. The first technique exploits the method of the "heated and non-heated wires" and is validated on wet clay by comparison with previous works [Mounanga et al., Eur. Phys. J. Appl. Phys. 26, 65 (2004)]. It is then used to quantify the evolution of both thermal conductivity and volumetric heat capacity of hardening cement pastes maintained at $294 \pm 1 \mathrm{~K}$. The second technique is based on the classical method of the "heating film" and a data treatment using forward calculation. This technique is first used to measure the properties of wellknown materials (hardened mortars, wet sand [Mounanga et al., Eur. Phys. J. Appl. Phys. 26, 65 (2004); Delacre, Ph.D. thesis, University of Artois, 2000] and glass [Bastian, Rev. Phys. Appl. 22, 431 (1987)] and then applied to media whose properties evolve both over time and through space (drying sand).
\end{abstract}

\section{Introduction}

In the field of Civil Engineering, the materials either natural (wood, rock) or artificial (concrete, mortar) are often multiphase materials. Some are partially water-saturated porous media and exhibit complex behaviour when exposed to chemical, mechanical or physical solicitations. In particular, building materials can undergo temperature gradients during their lifetime, which can alter their mechanical or aesthetic performances by generating cracks. Predicting the thermomechanical behaviour of the structure $[4-6]$ requires the determination of the material thermophysical properties.

The present paper describes the theoretical bases, the development and the validation of two different measurement methods usable to determine the heat transfer parameters of evolutive materials (thermal conductivity and volumetric heat capacity).

The first technique is based on the method of the "heated and non-heated wires" (HnHW method). It is validated on wet clay and then applied to the study of hydrating cementitious matrices maintained at $294 \pm 1 \mathrm{~K}$ under endogenous conditions (no water exchange with the exterior) during the first 24 hours of hydration. A peripheral thermal regulation system enables to maintain the matrix

\footnotetext{
a e-mail: pierre.mounanga@univ-nantes.fr
}

in quasi-constant temperature conditions since the hydration heat release can perturb the measurement [1]. The theoretical bases of the HnHW method consider the transient temperature field around a continuous line source dissipating a constant power in an infinite medium. The fact that the material sample is a finite medium demands to correct the temperature measurement before further treatment. This correction is evaluated by means of the image method.

The second technique is adapted from that of the "heating film". It allows, thanks to a result analysis based on inverse treatment, to study materials that do not release heat and whose properties evolve over time and through space. Teledeltos paper strips play the role of the heating film. This experimental method and the use of Teledeltos paper allow a short time of measurement and thus a weak disturbance of the medium. Temperature profiles, recorded at different locations inside the medium, are used as input data for a forward calculation program, exploiting a minimization function implemented in MatLab ${ }^{\circledR}$ (MathWorks inc.). The "heating film" method coupled with inverse calculation, noted HFIC, is first validated on reference materials (hardened cement pastes and mortars). Then the functions describing the spatial evolutions of the thermal properties of wet sand submitted to drying are determined. 
Before presenting the experimental devices, a brief reminder of the theoretical background of heat and mass transfers in porous media is exposed in Section 2.

\section{Heat and mass transfers in porous media}

Let us consider a non-deformable medium whose porosity contains liquid water, vapour and air. Assuming the following hypothesis:

- constant and uniform pressure of the gaseous phase in the material,

- slow changes in the material microstructure as compared with the time of observation $(<5$ minutes in the present study),

- weak rate of chemical reactions,

- radiative heat transfer neglected,

- heat of wetting neglected,

- hysteresis effects neglected,

heat and mass transfers can be modelled by the following set of equations [7]:

$$
\left\{\begin{array}{l}
\rho C_{P} \frac{\partial T}{\partial t}=\vec{\nabla} \cdot\left(\lambda \vec{\nabla} T+\rho_{l} L_{v} D_{\theta v} \vec{\nabla} \theta\right) \\
\frac{\partial \theta}{\partial t}=\vec{\nabla} \cdot\left(D_{\theta} \vec{\nabla} \theta+D_{T v} \vec{\nabla} T\right)+\frac{\partial K_{l}}{\partial z}
\end{array}\right.
$$

$-T$ : temperature $[\mathrm{K}]$

$-\theta$ : water content $[\%]$

- $t$ : time $[\mathrm{s}]$

$-z$ : ascending vertical coordinate $[\mathrm{m}]$

$-\rho C_{P}$ : volumetric heat capacity $\left[\mathrm{J} \mathrm{m}^{-3} \mathrm{~K}^{-1}\right.$

$-\lambda$ : apparent thermal conductivity $\left[\mathrm{W} \mathrm{m}^{-1} \mathrm{~K}^{-1}\right]$

$-L_{v}$ : latent heat of water vaporisation $\left[\mathrm{J} \mathrm{kg}^{-1}\right]$

$-D_{\theta}$ : global hydric diffusivity $\left[\mathrm{m}^{2} \mathrm{~s}^{-1}\right]$

- $D_{T v}$ : vapour diffusivity due to temperature gradient $\left[\mathrm{m}^{2} \mathrm{~s}^{-1}\right]$

- $D_{\theta v}$ : vapour diffusivity due to water content gradient $\left[\mathrm{m}^{2} \mathrm{~s}^{-1}\right]$

- $K_{l}$ : hydraulic conductivity $\left[\mathrm{m} \mathrm{s}^{-1}\right]$

$-\rho_{l}$ : density of the liquid $\left[\mathrm{kg} \mathrm{m}^{-3}\right]$.

Assuming that moisture diffusion is a much slower process than heat diffusion, it is possible to develop fast transient measurement methods that do not lead to modifications in the water content [8]. In this case, the heat flux $-\rho_{l} L_{v} D_{\theta v} \nabla \vec{\theta}$ is much lower than $-\lambda \nabla \vec{T}$ and system 1 can thus be simplified in:

$$
\rho C_{P} \frac{\partial T}{\partial t}=\vec{\nabla} \cdot(\lambda \vec{\nabla} T)
$$

where $\lambda=\lambda_{0}+\rho_{l} L_{v} D_{T v}$, the "apparent" thermal conductivity takes into account the pure heat conduction phenomenon described by Fourier's law $\left(\lambda_{0}\right.$ : medium pure thermal conductivity $\left[\mathrm{W} \mathrm{m}^{-1} \mathrm{~K}^{-1}\right]$ ) and the internal evaporation-condensation mechanism due to the diffusion of water vapour inside the pores and induced by temperature gradients [9].

\section{Presentation of the experimental methods}

The experimental determination of wet porous media thermal properties is a complex problem. This complexity is related to the state of pore water: it is important to ensure that the measurement method does not induce internal movements of the liquid phase and/or liquid/vapour phase change. Those phenomena can indeed have a significant influence on heat transfers and consequently on the thermophysical properties of the material $[9,10]$. Transient methods are thus preferred because they allow to shorten the test duration and therefore to avoid internal moisture movements [11]. In these methods, the heat transfer parameters are determined from the temperature response of a sample submitted to a short thermal solicitation. In this paper, two techniques are used:

- the "heated and non-heated wire" method (HnHW),

- the "heating film" method (HF).

\subsection{HnHW method}

\subsubsection{Experimental device}

In this measurement method, the follow-up of the temperature evolution of two stainless-steel wires, whose one is heated during a short time lapse, makes it possible to determine the thermal conductivity and the volumetric heat capacity of the surrounding medium. The medium temperature must be initially uniform. In order to fulfil this condition, the assembly is equipped with a system of temperature control. In the case of hydrating cementitious matrices, this system eliminates the influence of the hydration heat and ensures that the material properties evolve only over time.

Figure 1 shows a schematic diagram of the experimental device. The assembly is composed of a rigid, parallelepipedal mould, whose walls are covered with a $50 \mathrm{~mm}-$ thick layer of thermal insulator (polyurethan foam) (see Fig. 1). The stainless-steel wire-sensors, covered with a thermo-retractable sheath, are tightened between two rigid elements situated at both ends of an aluminium rule embedded in the bottom of the mould. The sheath prevents electric conduction effect between the wires and the wet material. Bastian and Khelidj [12] showed that the influence of the thermo-retractable sheath on experimental values of $\lambda$ and $\rho C_{P}$ can be neglected.

The material is cast on the higher face of the first water circulation box placed at the bottom of the mould. The second water circulation box is then placed on the top of the sample. The weight of this box is transferred on the peripheral insulator layer through the outlet and inlet pipes of the water circuit, avoiding the crushing of the sample.

A thermocouple is embedded in the medium in order to follow the temperature evolution.

At the end of casting, the electric circuit providing the power to the heated wire is closed. This electric circuit is shown at Figure 2. It is composed of two meshes. During the heating phase $\left(t_{h}=120 \mathrm{~s}\right)$, an alternative current 


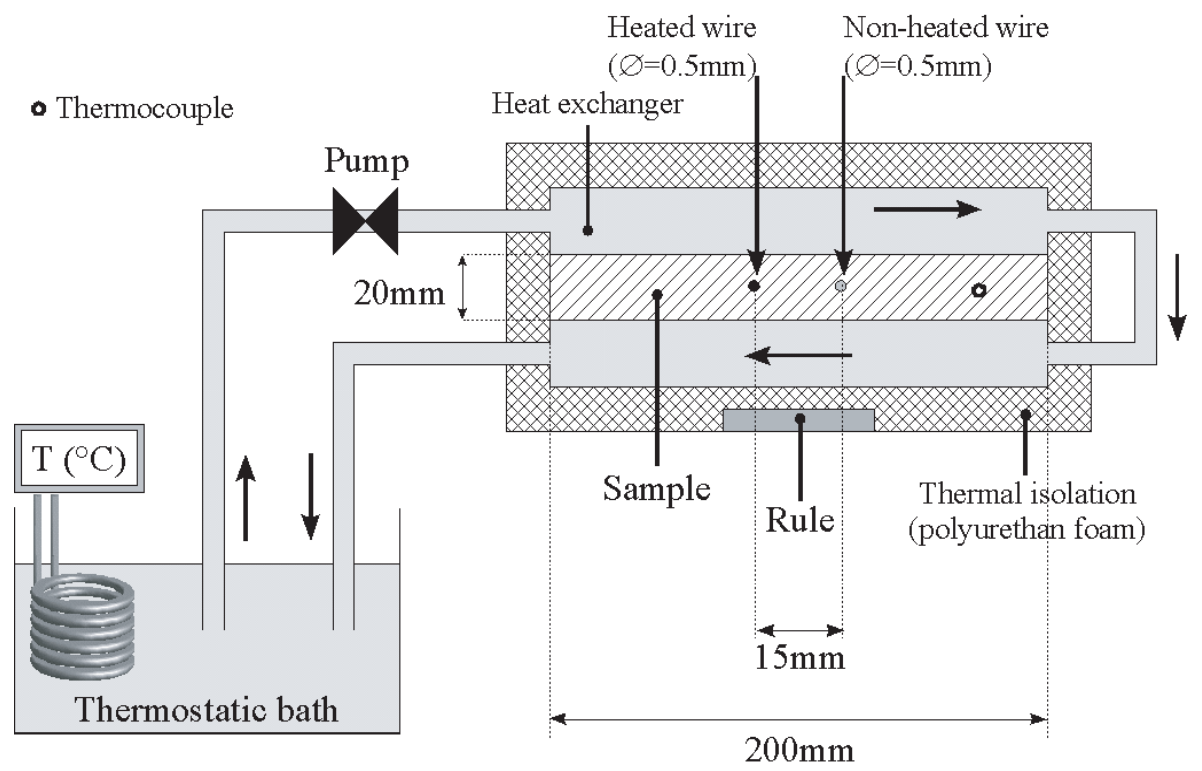

Fig. 1. Schematic diagram of the "heated and non-heated wire" device (HnHW method).

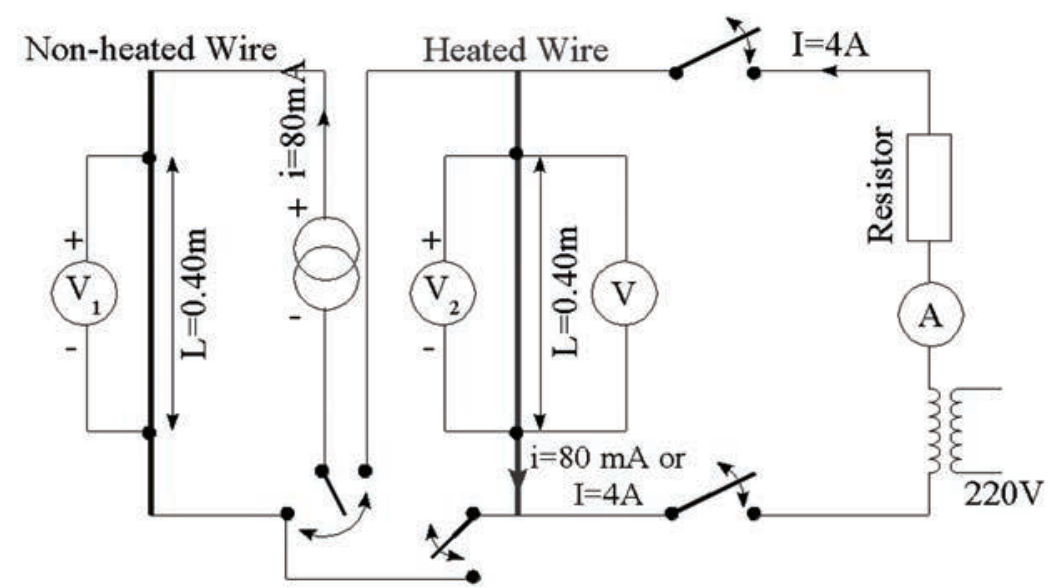

Fig. 2. Diagram of the electric circuit for "the heated and non-heated wire" device.

$(\mathrm{AC} ; I=4 \mathrm{~A})$ passes through the first mesh. Taking into account the stainless-steel wire resistance, this current delivers an electric power of $20 \mathrm{~W} \mathrm{~m}^{-1}$. A direct current (DC; $i=80 \mathrm{~mA}$ ), provided by a stabilized power supply passes continuously through the second mesh. The DC, too weak to overheat the stainless-steel wire-sensors, enables to measure their electric voltage. A relay, controlled by a data acquisition station, allows the closing of the first mesh during the heating phase and the closing of the second mesh during the measurement phase. The data acquisition station records automatically the evolutions of the stainless-steel wire electric voltage and the embedded thermocouple signal. The observation time is 3 minutes and the measurement cycle period is 15 minutes. The first measurement cycle is carried out 15 minutes after casting.

The knowledge of the "resistance vs. temperature" law of the stainless-steel wires makes it possible to convert the electric voltage evolution into temperature evolution.

\subsubsection{Theoretical description}

The HnHW method can be used to determine the thermophysical properties of a material from the temperature time evolution of wires embedded in the medium $[12,13]$.

\subsubsection{Continuous source line in an homogeneous infinite medium}

Let us consider a continuous source line, materialized by an infinitely thin wire, dissipating a constant linear power $P_{l}\left[\mathrm{~W} \mathrm{~m} \mathrm{~m}^{-1}\right]$ from the moment $t=0$ to $t_{h}$, in an infinite medium whose temperature is initially uniform. This medium is characterized by its thermal conductivity $\lambda\left[\mathrm{W} \mathrm{m}^{-1} \mathrm{~K}^{-1}\right]$ and its volumetric heat capacity $\rho C_{P}\left[\mathrm{~J} \mathrm{~m}^{-3} \mathrm{~K}^{-1}\right]$. In these conditions, the heat conduction equation is:

$$
\rho C_{P} \frac{\partial T}{\partial t}=\vec{\nabla} \cdot(\lambda \vec{\nabla} T)
$$


The resolution of equation (3) in cylindrical coordinates gives the following relation:

$$
T(r, t)=\frac{P_{l}}{4 \pi \lambda} E_{1}\left(\frac{r^{2}}{4 a t}\right)
$$

where $r$ is the distance to the source $[\mathrm{m}], a \quad\left(=\frac{\lambda}{\rho C_{P}}\right)$ the medium thermal diffusivity $\left[\mathrm{m}^{2} \mathrm{~s}^{-1}\right]$ and $t$ the time $[\mathrm{s}]$. $E_{1}(x)$ is the exponential integral of $x$ :

$$
E_{1}(x)=\int_{x}^{+\infty} \frac{e^{-v}}{v} d v
$$

Equation (4) gives the temperature evolution of a nonheated wire embedded in the material, parallel to the heated wire at a distance $r$, but also describes the thermal evolution of the heated wire [14]. As soon as heating is stopped, the temperature has to be calculated from the following relation:

$$
T(r, t)=\frac{P_{l}}{4 \pi \lambda}\left[E_{1}\left(\frac{r^{2}}{4 a t}\right)-E_{1}\left(\frac{r^{2}}{4 a\left(t-t_{h}\right)}\right)\right]
$$

This latter is obtained from the superimposition of the temperature field relative to the heat source and the temperature field of a heat sink.

The temperature evolution of the heated and nonheated wires from $t=0$ to $t>t_{h}$ can be seen in Figures 19 and 20 respectively (Appendix A). For the heated wire and for $t>>t_{h}$, equation (6) can be approximated by $[15]$ :

$$
T(r, t)=\frac{P_{l}}{4 \pi \lambda}\left[\ln \left(\frac{4 a t}{r^{2}}\right)-\ln \left(\frac{4 a\left(t-t_{h}\right)}{r^{2}}\right)\right]
$$

which yields, after transformation:

$$
T(r, t)=\frac{P_{l}}{4 \pi \lambda} \ln \left(\frac{t}{t-t_{h}}\right)
$$

$\lambda$ can thus be written as (where $\Delta$ represents a time variation between two points of the curve, see Fig. 3):

$$
\lambda=\frac{P_{l}}{4 \pi} \frac{\Delta\left(\ln \frac{t}{t-t_{h}}\right)}{\Delta T}
$$

For the non-heated wire, the expression of the theoretical temperature curve slope at point I, indicated in Figure 20 (Appendix A) [13], can be derived from equation (4).

The development of the exponential integral for very small values of $x$ is [15]:

$$
E_{1}\left(\frac{1}{x}\right) \approx \frac{x}{e^{1 / x}}\left(\frac{1+A_{1} x+A_{2} x^{2}}{1+B_{1} x+B_{2} x^{2}}\right) \approx \frac{x}{e^{1 / x}}
$$

At the point of inflexion I (see Fig. 4), where $\frac{4 a t_{\mathrm{I}}}{r^{2}}=1$, equation (4) can thus be written:

$$
T\left(r, t_{\mathrm{I}}\right)=\frac{P_{l}}{4 \pi \lambda} \frac{4 a t_{\mathrm{I}}}{e r^{2}}
$$

with $e=2.718 \ldots$ and finally:

$$
\rho C_{P}=\frac{P_{l}}{e \pi r^{2}}\left(\frac{\Delta t}{\Delta T}\right)_{\mathrm{I}}
$$

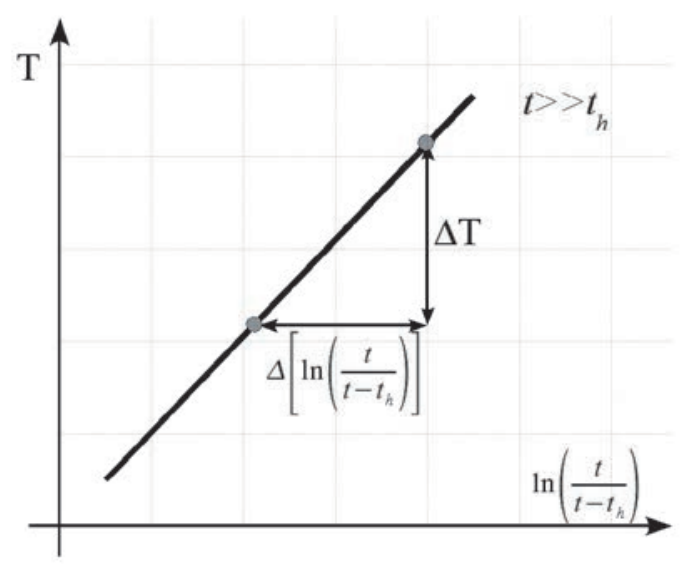

Fig. 3. Temperature vs. non-dimensional time for the heated wire during the post heating phase.

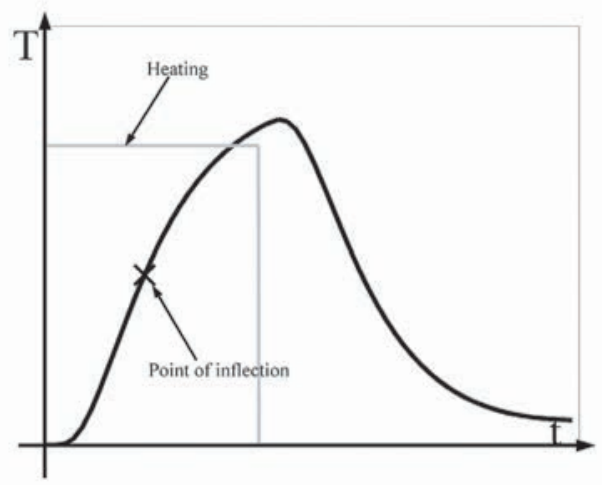

Fig. 4. Temperature vs. time for the non-heated wire.

\subsubsection{Continuous source line in a finite medium - Image} method

In the case of a finite medium, equations (3-11) can not be applied directly. Considering a slab, for example, the boundary conditions on its upper and lower faces influence the time evolution of the wire temperature. Therefore, the determination of the thermal properties from equations $(9$, 11) lays on a preliminary correction of the temperature profiles: the influence of the boundary conditions is quantified by the "image method" [16].

According to this method, the real configuration, shown in Figure 5a, is equivalent to the configuration in an infinite space, given in Figure 5b.

In the second configuration (Fig. 5b), the problem finally consists in determining the temperature change of the heated and non-heated wires submitted to the influence of a heat source (heated wire) and to that of an infinity of alternated heat sinks and heat sources (imagewires), in an infinite medium, with an uniform initial temperature field.

Prior to the application of equations $(9,11)$ for the calculation of $\lambda$ and $\rho C_{P}$, the thermal effects of the image heat sources and sinks have to be quantified and subtracted from the temperature time evolution of the wires. 


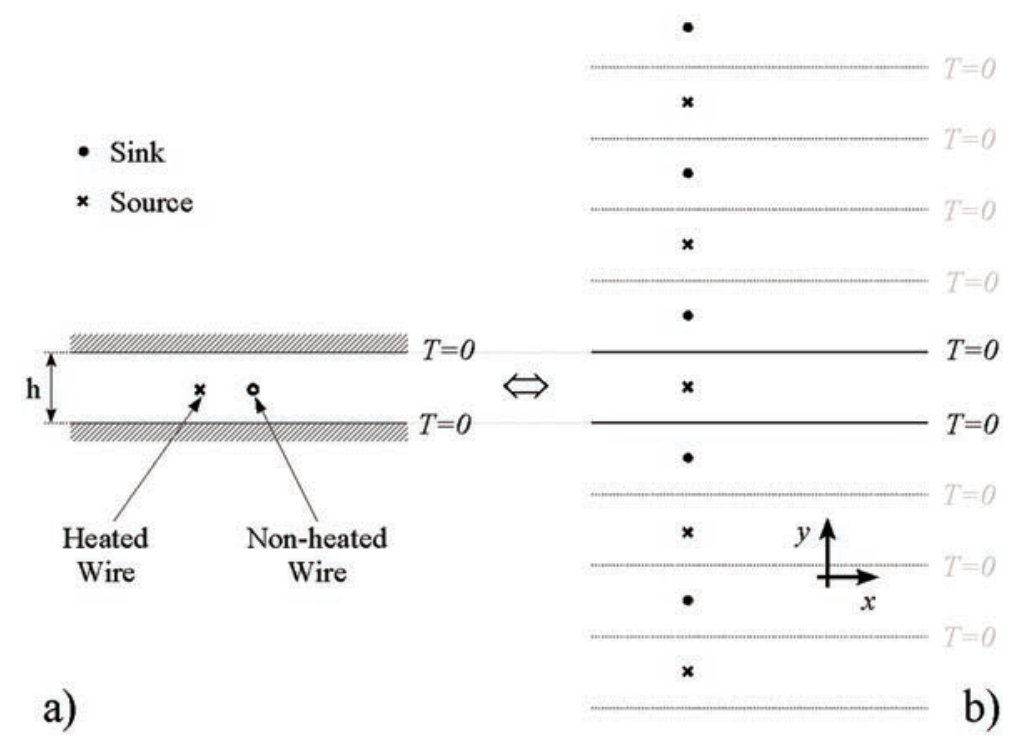

Fig. 5. Image method applied to the HnHW method.

Complete development of the "image method" is given in Appendix A.

\subsection{HF and HFIC methods}

\subsubsection{Experimental device}

The geometry of study is composed of parallelepipedal test samples of square section.

The sample receives a heat flux $\Phi\left[\mathrm{W} \mathrm{m}{ }^{-2}\right]$ through one of its faces. The other faces are thermally insulated.

For materials with uniform thermophysical properties, only two thermocouples, centred on the heating film and on the opposite insulated face are sufficient to determine $\lambda$ and $\rho C_{P}$ (HF method - [1]). Analytical relations indeed link the difference between the temperature of the two thermocouples to $\lambda$ and the slope of the temperature time evolutions to $\rho C_{P}$.

In the case of materials with non-uniform properties (for example drying materials exhibiting internal water content gradients) no analytical equation is available to determine the functions describing these parameters. The thermocouples are then placed into the material at different locations and the temperature time evolutions are used as input data for the forward calculation program. The experimental device for the HFIC method is shown in Figure 6 and examples of temperature time evolutions are presented in Figure 7. In the experiments, the temperature increase is always lower than $4 \mathrm{~K}$.

\subsubsection{Theoretical description}

Temperature profiles measured at different locations in the material are analysed with an inverse calculation program to determine the medium thermal properties. Forward calculation lays on the numerical resolution of the heat transfer equation (Eq. (2)). The thermophysical parameters leading to the same time evolutions of temperature are sought numerically. This method is particularly adapted when space variations of the thermophysical properties are observed, especially during the drying of materials.

\subsubsection{Direct problem}

Heat conduction equation together with the thermophysical properties of the material, the initial and boundary conditions can give the temperature field in a material. In order to solve the problem numerically, the partial derivative equations are discretized applying central difference approximation and the ADI method (Alternating Direction Implicit - [17]). A system of linear equations is then built in the shape of two easily inversible tridiagonal matrices (see Appendix B).

For two-dimensional problems, ADI method consists in using an implicit scheme in one direction of space and an explicit one in the perpendicular direction at one time step, and then inverting the implicit and explicit directions at the next time step.

Flux boundary conditions are written using "fictitious nodes" [17]. The temperature derivatives are then expressed as the difference between a temperature at a node inside the material and a temperature at a virtual node out of the material.

The ADI method yields the following systems of equations:

$A_{x} T^{2 n}=B_{z} T^{2 n-1}+C_{z}$ for the even moments (explicit in $z$ and implicit in $x$ )

$A_{z} T^{2 n+1}=B_{x} T^{2 n}+C_{x}$ for the odd moments (explicit in $x$ and implicit in $z$ )

where $A_{x}, A_{z}, B_{x}$ and $B_{z}$ are tridiagonal matrices and $C_{x}$ and $C_{z}$ are vectors expressing the boundary conditions. Thus, the temperature field can be obtained by calculating 


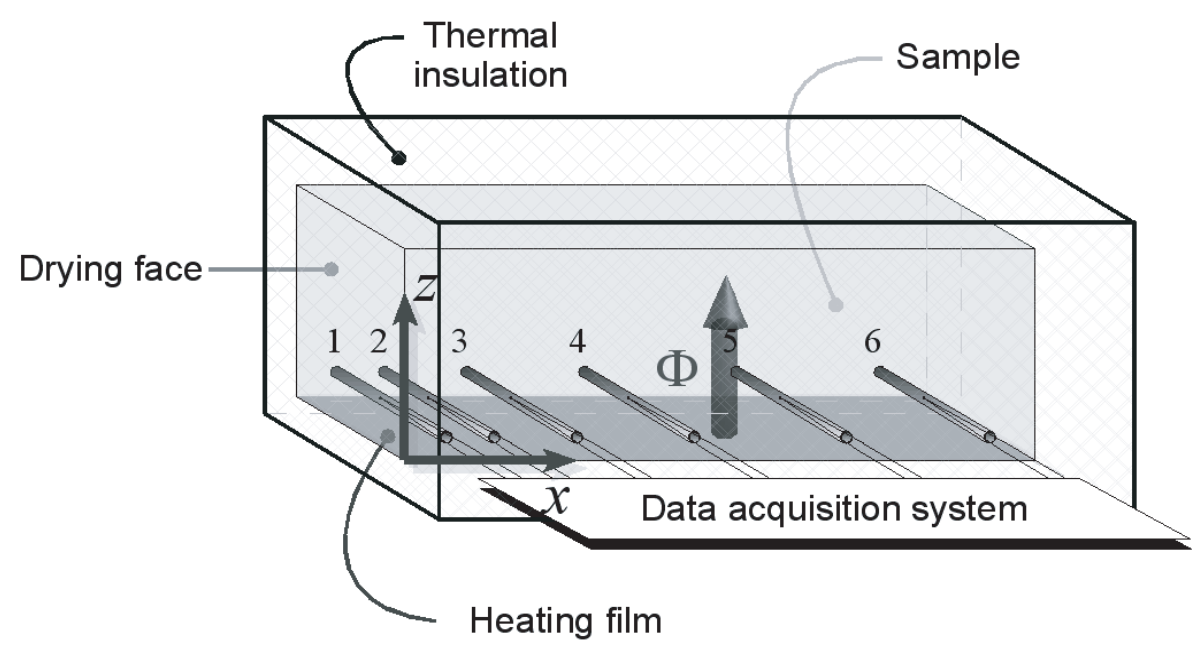

Fig. 6. Schematic view of the experimental device for the HFIC method.

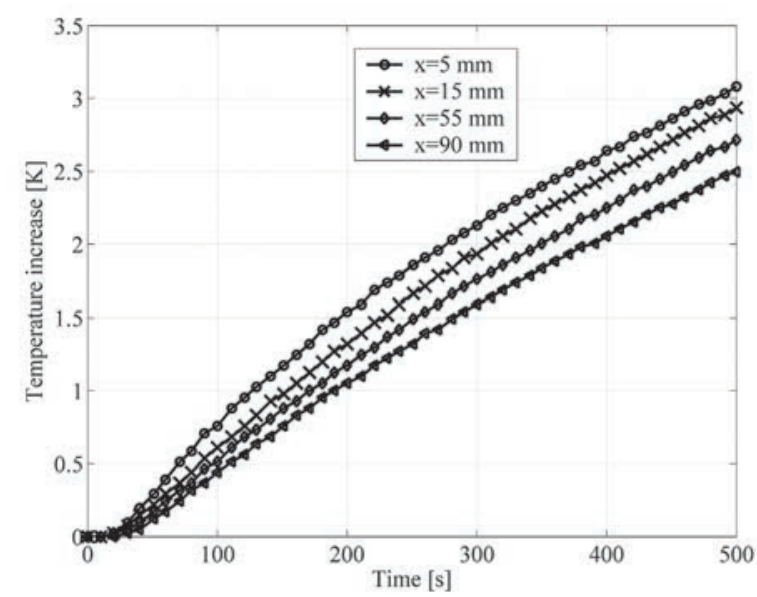

Fig. 7. Example of temperature time evolutions measured by thermocouples placed at different locations in a medium with non-uniform properties.

the inverse matrices of $A_{x}$ and $A_{z}$ :

$$
\begin{aligned}
& T^{2 n}=A_{x}^{-1}\left(B_{z} T^{2 n-1}+C_{z}\right) \\
& T^{2 n+1}=A_{z}^{-1}\left(B_{x} T^{2 n}+C_{x}\right)
\end{aligned}
$$

\subsubsection{Inverse problem}

The resolution requires successive computations of the direct problem. Test function has to be minimized, i.e. the difference between the measured experimental data and the calculated numerical data has to be made as small as possible. Minimization is done by the conjugate gradient method [18]. In this work, the test function has the following form:

$$
\varepsilon_{i}\left(\lambda_{i} ;\left(\rho C_{P}\right)_{i}\right)=\sum_{t=0}^{t=t_{\max }} \sum_{n=1}^{N}\left(T_{n, t}^{\exp }-T_{n, t}^{n u m}\left(\lambda_{i} ;\left(\rho C_{P}\right)_{i}\right)\right)^{2}
$$

where $t$ is the discrete time $[\mathrm{s}], t_{\max }$ the observation time $[\mathrm{s}], N$ the number of temperature profiles [-], $T_{n, t}^{\exp }$ the experimental temperature profile $[\mathrm{K}], T_{n, t}^{\text {num }}$ the computed temperature profile $[\mathrm{K}]$.

Figure 8 shows the principle of the inverse method. Initial values are given to the thermal conductivity and to the heat capacity and constitute the initial guesses of the problem. Those parameters are used to calculate the temperature field and to obtain a first estimate of the test function from a comparison with the experimental data (Eq. (12)). Calculation of the temperature field for parameters close to the initial guesses makes it possible to know the local shape of the test function and to guide the research towards the optimal parameters. Computation is terminated as soon as the difference between two consecutive values of $\varepsilon_{i}\left(\lambda_{i} ;\left(\rho C_{P}\right)_{i}\right)$ becomes lower than $10^{-6}$.

\section{Experiments}

\subsection{Studied materials}

\subsubsection{Cement}

The cement used during the tests is CPA-CEM I 52.5 PM ES from Le Teil (France). Its chemical composition is given in Table 1 . The characterization tests were carried out in the section "Characterization and Study of Materials" at the LCPC (Laboratoire Central des Ponts et Chaussées, Paris).

\subsubsection{Clay}

Clay is used to validate the HnHW method. It is chosen because of its consistency and its thermophysical characteristics close to those of fresh cement pastes: it is a homogeneous sample of natural clay, already studied by Bastian [19]. 


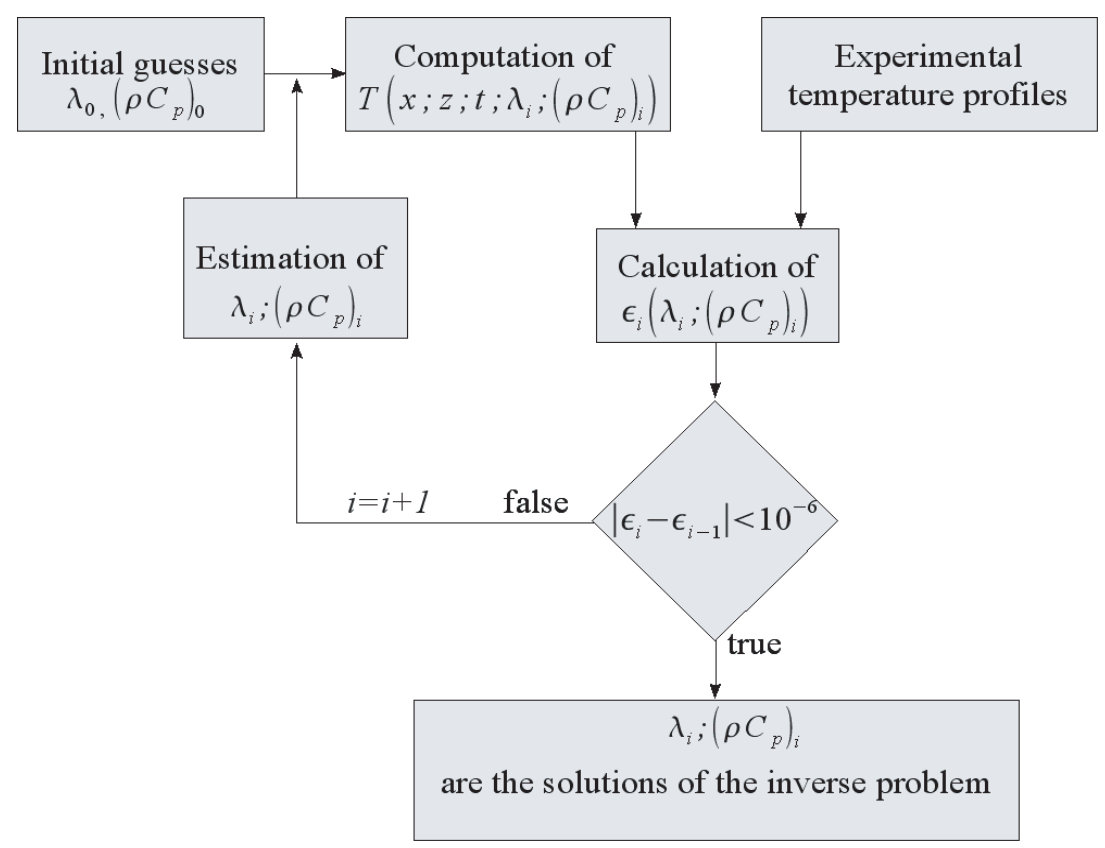

Fig. 8. Principle of the inverse method.

Table 1. Chemical composition of the cement.

\begin{tabular}{|c|c|c|c|c|c|c|c|c|c|}
\hline & & Blaine : & $\begin{array}{l}\text { SPA-CE } \\
\text { ecific s! }\end{array}$ & $\begin{array}{l}\text { I I } 52.5 \\
\text { face are }\end{array}$ & $\begin{array}{l}\text { M ES } \\
3320 \\
\end{array}$ & $\mathrm{n}^{2} \mathrm{~g}^{-}$ & & & \\
\hline $\begin{array}{c}\text { Composition } \\
\% \text { (mass) }\end{array}$ & $\begin{array}{l}\mathrm{CaO} \\
66.39\end{array}$ & $\begin{array}{l}\mathrm{SiO}_{2} \\
21.17\end{array}$ & $\begin{array}{c}\mathrm{Al}_{2} \mathrm{O}_{3} \\
2.69\end{array}$ & $\begin{array}{c}\mathrm{Fe}_{2} \mathrm{O}_{3} \\
1.96\end{array}$ & $\begin{array}{l}\mathrm{MgO} \\
0.65\end{array}$ & $\begin{array}{l}\mathrm{SO}_{3} \\
2.43\end{array}$ & $\begin{array}{c}\mathrm{NaO}_{2} \\
0.3\end{array}$ & $\begin{array}{l}\mathrm{K}_{2} \mathrm{O} \\
0.22\end{array}$ & $\begin{array}{l}\mathrm{CO}_{2} \\
1.56\end{array}$ \\
\hline
\end{tabular}

Saturated clay is mixed during fifteen minutes. Distilled water is gradually added in order to obtain desired consistency. An impermeable plastic film is then placed on the mixer bowl. Drying is thus prevented during the few minutes that separate the end of mixing and the casting into the experimental device. The clay water content, defined as the mass ratio between water and dry material, is $123 \%$.

\subsubsection{Cement pastes and mortars}

Cement pastes and mortars are prepared by first mixing the solid phase during thirty seconds in order to break the cement agglomerates and to homogenize the sand-cement mixture. Distilled and deaerated water is then added. Mixing is carried out during 3 minutes using a 5 L-mixer.

\subsubsection{Sand}

The sand comes from the region of Nantes (France), it has been sieved to keep the particles between $80 \mu \mathrm{m}$ and $1 \mathrm{~mm}$.

\subsection{HnHW method - Calibration of the stainless-steel wires}

The stainless-steel wires, embedded in the material, are used both as heating element and thermal sensors: their electric resistance $R$ is indeed linearly related to their temperature $T$ (Fig. 9) and can be modelled as:

$$
R(T)=R_{0}(1+\alpha T)
$$

where $R_{0}$ is the resistance at $0{ }^{\circ} \mathrm{C}[\Omega]$ and $\alpha$ is the temperature coefficient $\left[\mathrm{K}^{-1}\right]$.

A calibration is carried out in a thermostated bath in order to determine $\alpha$. The detailed method can be found in [12]. For the actual stainless-steel wire, the following value of $\alpha$ is obtained:

$$
\alpha=2.6304 \times 10^{-3} \mathrm{~K}^{-1}
$$

Knowing the material temperature that is presumed uniform before heating the wire, voltage measurements provide, with a good accuracy, the temperature of the wires [12].

\subsection{HFIC method - Characteristics of the heating film}

Teledeltos paper is used as the heating film. Its properties have already been given in [1]. The application of an electric potential difference between two points of a Teledeltos paper sheet creates an electric current able to heat the surface by Joule effect. In order to check the uniformity of heating on the paper sheet surface, a $50 \mathrm{~V}$-electric potential difference is applied at the boundaries of the 


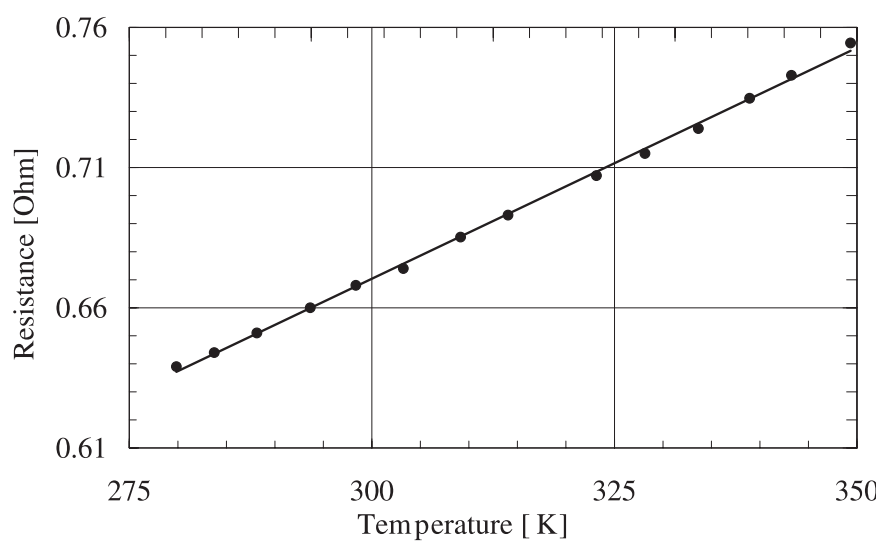

Fig. 9. Calibration curve of the stainless-steel wire.

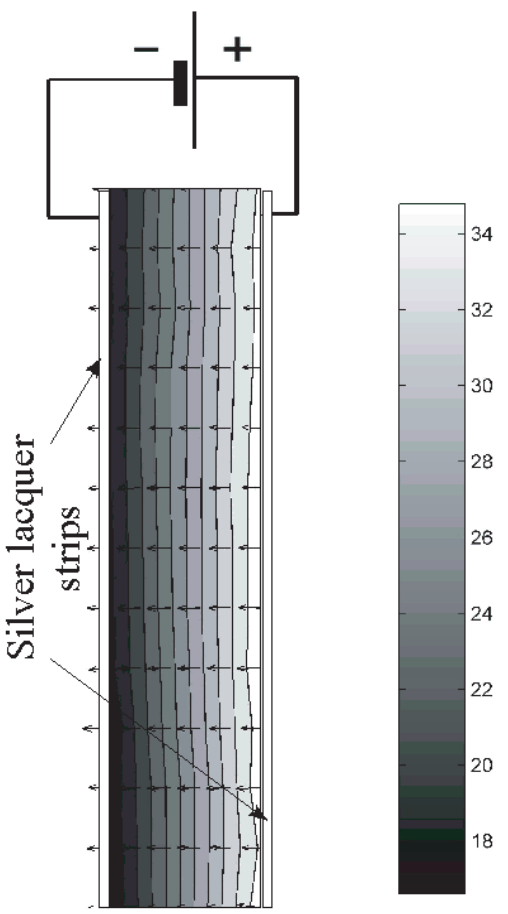

Fig. 10. Experimental electric potential field $[\mathrm{V}]$ at the surface of the heating film.

paper, painted with silver lacquer. This potential difference induces the circulation of a $50 \mathrm{~mA}$-current through the film. The power dissipated by the film is then $2.5 \mathrm{~W}$, which corresponds to a heat flux of $280 \mathrm{~W} \mathrm{~m}^{-2}$. From voltage measurements in various points on the film surface, equipotential lines can be drawn. These lines are vertical, the power is thus uniformly dissipated on the whole surface of the heating film (see Fig. 10). Moreover, temperature measurements have proved that the power is only dissipated by the unpainted surface.

\subsection{Validation tests}

HF method, described by Mounanga et al. [1] and already validated on glass plates, is used as a reference technique.
The validation of the HnHW and HFIC methods consists in comparing the thermal conductivity and volumetric heat capacity values of the same material obtained from the different methods.

\subsubsection{HnHW method}

Two tests on wet clay are carried out with the classical $\mathrm{HF}$ technique and three tests with the $\mathrm{HnHW}$ technique. Each test is achieved with a new sample.

\subsubsection{HFIC method}

The tested materials are on one hand four 60 day-aged mortars with different water-to-cement mass ratios $(W / C$ ratios) and sand-to-cement mass ratios $(S / C$ ratios), and on the other hand glass and wet sand (mass water content: $w=10 \%$ ). From casting to test, the mortars are kept in endogenous conditions in order to have their properties be uniform.

During the test, the samples are equipped with two thermocouples. One is in contact with the heating film; the second one is placed on the opposite insulated face.

The properties determined by the HF method are averages of four successive tests. For the inverse method, initial guesses of $1 \mathrm{~W} \mathrm{~m}^{-1} \mathrm{~K}^{-1}$ for $\lambda$ and of $3 \times 10^{6} \mathrm{~J}$ $\mathrm{m}^{-3} \mathrm{~K}^{-1}$ for $\rho C_{P}$ are taken.

\subsection{Application to evolutive porous materials}

\subsubsection{HnHW method - Hardening cement pastes}

In massive concrete structures, the hydration heat release of the cementitious matrix generates thermal stresses high enough to increase the risk of premature cracking of concrete. In most of the models proposed to predict the thermomechanical behaviour of concrete at early age, the thermophysical properties are considered as constant parameters, but very few studies [20,21] have been achieved to assess that hypothesis.

Experimental tests are performed to measure the thermal conductivity and the volumetric heat capacity of various $W / C$ ratio cement pastes, during the first twenty four hours of hydration. The aim of the tests is to quantify the possible variations of $\lambda$ and $\rho C_{P}$ due to the microstructural changes occurring during the setting period of the matrices.

\subsubsection{HFIC method - Drying sand}

Tests are carried out on drying wet sand prismatic samples $\left(4 \times 4 \times 16 \mathrm{~cm}^{3}\right)$ using the HFIC method. The initial water content of the sample is $10 \%$. Only the upper square face is in contact with the air and allows moisture exchanges. Before commencing the test, drying is performed in a room at a temperature of $294 \mathrm{~K}$ and a relative humidity of $55 \%$ 
Table 2. Comparison between the experimental results obtained with HF and HnHW techniques.

\begin{tabular}{c|ccc|cccc}
\hline Wet clay & \multicolumn{3}{|c|}{ HF method } & \multicolumn{4}{c}{ HnHW method } \\
(water content: 123\%) & Test No. 1 & Test No. 2 & Mean value & Test No. 1 & Test No. 2 & Test No. 3 & Mean value \\
\hline$\lambda\left[\mathrm{W} \mathrm{m}^{-1} \mathrm{~K}^{-1}\right]$ & 0.91 & 0.93 & 0.92 & 0.88 & 0.90 & 0.90 & 0.89 \\
$\rho C_{P}\left[10^{6} \mathrm{~J} \mathrm{~m}^{-3} \mathrm{~K}^{-1}\right]$ & 3.95 & 3.74 & 3.85 & 4.07 & 3.96 & 3.93 & 3.99 \\
\hline
\end{tabular}

Table 3. Comparison between the experimental results obtained by HF and HFIC methods.

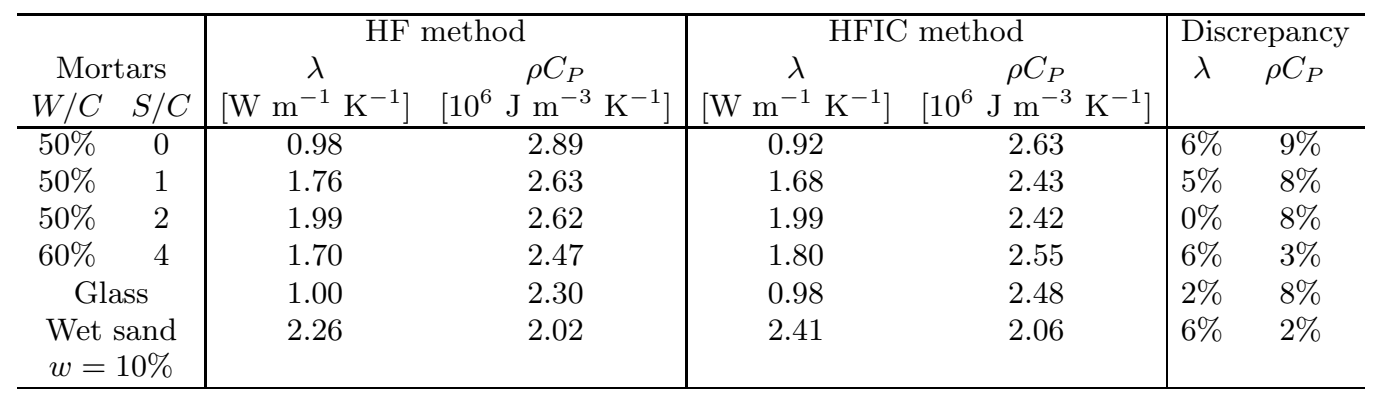

during 3 days. As the thermal properties evolve with the distance $x$ to the drying face, the inverse method is chosen to identify functions $\lambda(x)$ and $\rho C_{P}(x)$. The water content $w(x)$ is also measured. It is assumed to be uniform in a cross section of the sample located at $x$.

The water content is measured by a classical method described hereafter. Slices are cut in the sample and placed into different flasks. Each slice is then heated in a microwave oven and weighted until the mass is constant. The difference between the initial and final sand slice masses makes it possible to calculate its water content $w$ :

$$
w(\%)=\frac{M_{i}-M_{f}}{M_{f}-M_{\text {flask }}} 100 \%
$$

where $M_{\text {flask }}$ is the flask mass [g], $M_{i}$ is the initial mass of the flask filled with the wet sand $[\mathrm{g}]$ and $M_{f}$ the final mass of the flask filled with dried sand [g].

\section{Results and discussions}

\subsection{Validation of the experimental methods}

\subsubsection{HnHW method}

The experimental results are given in Table 2 .

A good repeatability of the measurements achieved with the HnHW device is observed: the relative difference between the measurements and the average value is lower than $2 \%$. Moreover, the results obtained from both methods are very close, the relative variations are lower than 3 and $4 \%$ for the thermal conductivity and for the volumetric heat capacity, respectively.

\subsubsection{HFIC method}

Table 3 presents the thermophysical property values measured by the HF method and calculated by the HFIC method.

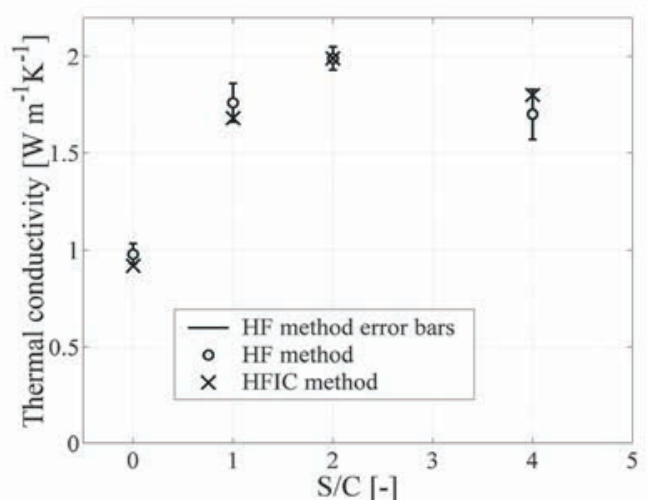

Fig. 11. Mortar thermal conductivity vs. $S / C$ ratio; comparison between the HF method and the HFIC method.

Figures 11 and 12 show the evolutions of $\lambda$ and $\rho C_{P}$ as a function of mortar $S / C$ ratio. The error bars are the standard deviations observed during the measurement by the HF method.

A good agreement between the data measured by the HF method and the inverse method can be noted. The values of $\lambda$ obtained by the inverse method are inside the zone of uncertainty of the HF method. The values of $\rho C_{P}$ determined by the inverse method are slightly lower than the HF method results. However, the agreement is satisfactory. A better estimation of the parameters is possible by refining the grid of calculation. Indeed, although the discretization scheme is unconditionally stable, the solution can evolve according to the coarseness of the grid.

\subsection{HnHW method - Hardening cement pastes}

\subsubsection{Temperature control of the samples}

Application of equations $(9,11)$ used for the HnHW method requires initial constant temperature before each 


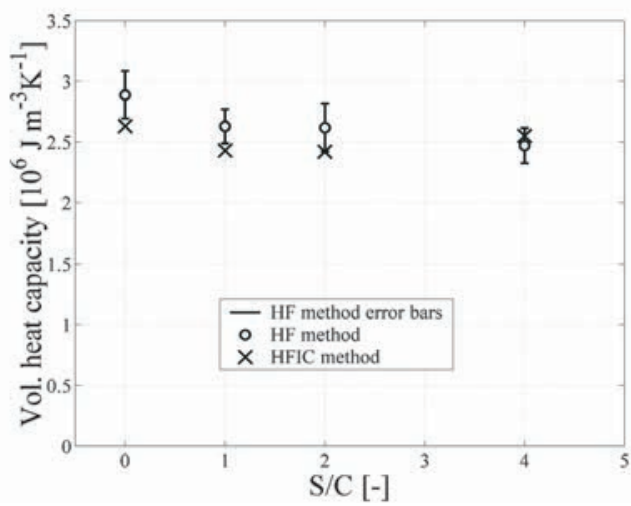

Fig. 12. Mortar volumetric heat capacity vs. $S / C$ ratio; comparison between the HF method and the HFIC method.

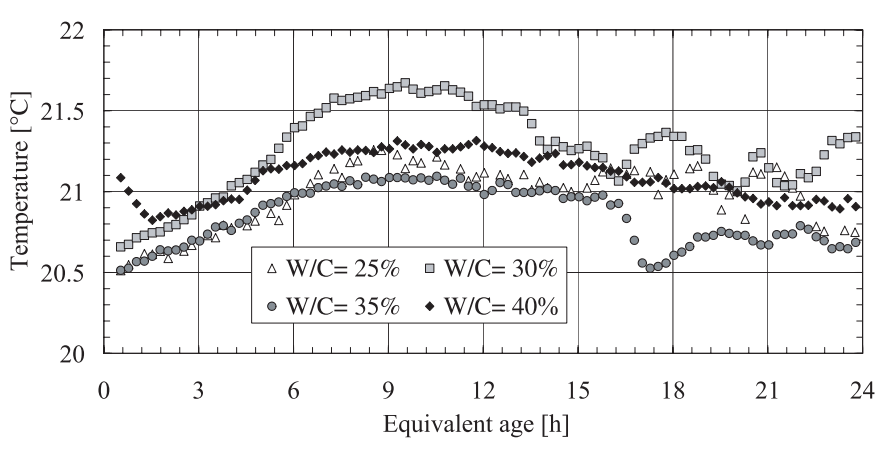

Fig. 13. Temperature of the cement paste samples during the tests.

measurement cycle. It is thus necessary to dissipate the heat generated by the cement hydration. With this aim, a peripheral system of thermal regulation is integrated into the experimental device (see Sect. 3.1.1).

A thermocouple is embedded in each sample in order to check the efficiency of this thermal regulation system (see Fig. 1).

Figure 13 reveals that, during the tests, the sample temperature is not constant; however, the thermal increase remains lower than $1 \mathrm{~K}$ and the maximum rate of the temperature evolution is lower than $0.3 \mathrm{~K} \mathrm{~h}^{-1}$. An elementary calculation shows that these variations have a negligible influence on the thermal evolution of the stainless-steel wires.

\subsubsection{Maturity and equivalent age}

The hydration of cement is a thermoactivated chemical process: it is accelerated by a high ambient temperature. It means that a cementitious matrix maintained at constant temperature follows a different "thermodynamic path" than the same material submitted to another thermal history. The concept of maturity has been introduced to take into account the effect of temperature on the cement hydration rate. This concept needs the knowledge of the real temperature history of the material and consists in transforming the real age $t$ of concrete into the equivalent age $t_{e q}$ using a suitable maturity function [22]. The

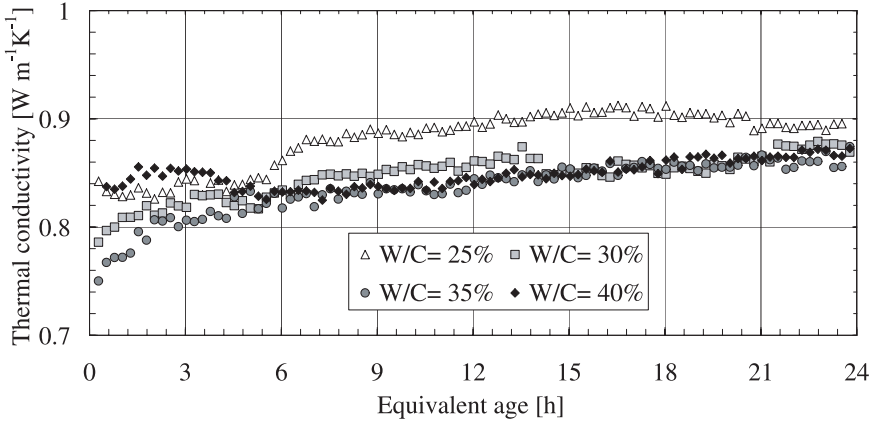

Fig. 14. Thermal conductivity of cement pastes with different $W / C$ ratios (test temperature: $294 \pm 1 \mathrm{~K}$ ).

maturity function suggested by Arrhenius is best qualified for a proper description. With this function, the equivalent age can be derived:

$$
t_{e q}=\int_{0}^{t} \exp \left(\frac{-E_{a}}{R}\left(\frac{1}{T(\tau)}-\frac{1}{T_{r e f}}\right)\right) d \tau
$$

with $E_{a}$ the apparent activation energy $\left[\mathrm{J} \mathrm{mol}^{-1}\right]$ and $R$ the perfect gas constant $\left[\mathrm{J} \mathrm{mol}^{-1} \mathrm{~K}^{-1}\right]$. In the following sections, the evolution of $\lambda$ and $\rho C_{P}$ of the cement pastes are expressed as a function of their equivalent age, considering the apparent activation energy of cement to be equal to $34.0 \mathrm{~kJ} \mathrm{~mol}^{-1}$ [23]. The reference temperature $T_{\text {ref }}$ is $293 \mathrm{~K}$, in agreement with European standard.

\subsubsection{Evolutions of $\lambda$ and $\rho C_{P}$ at $294 \pm 1 \mathrm{~K}$}

The experimental results obtained for the thermal conductivity and the volumetric heat capacity of early age cement pastes are shown in Figures 14 and 15.

During the first twenty-four hours, the thermal conductivity slightly increases (see Fig. 14), rising by 6,11 , 14 and $4 \%$ for the cement pastes with $W / C$ ratio $=25$, 30,35 and $40 \%$, respectively.

The influence of the $W / C$ ratio on the evolution of $\lambda$ remains weak. Indeed, the curves with $W / C$ ratio $=30,35$ and $40 \%$ are superimposed over almost the whole investigation period. Although the curve with $W / C$ ratio $=25 \%$ shows higher values, the differences with the other curves are lower than $7 \%$. Thermal conductivity values measured at 24 hours are close for the four $W / C$ ratios (see Tab. 4).

The volumetric heat capacity (Fig. 15) decreases during the first 24 hours of hydration. All the curves present the same decreasing evolution.

The high initial value of $\rho C_{P}$ can be explained by the presence of non-chemically bound water within the material. The solid phase has indeed a much lower volumetric heat capacity than "free" water [24]. The progressive consumption of water by the hydration reactions causes an increase of the solid phase volume fraction and indirectly a decrease in the volumetric heat capacity of the material.

The thermal diffusivity, noted $a$, also characterizes the thermal behaviour of a medium. 
Table 4. Thermophysical properties of cement pastes after 24 hours of hydration at $294 \pm 1 \mathrm{~K}$.

\begin{tabular}{ccccc}
\hline & $W / C=25 \%$ & $W / C=30 \%$ & $W / C=35 \%$ & $W / C=40 \%$ \\
\hline$\lambda\left[\mathrm{W} \mathrm{m}^{-1} \mathrm{~K}^{-1}\right]$ & 0.90 & 0.87 & 0.86 & 0.87 \\
$\rho C_{P}\left[10^{6} \mathrm{~J} \mathrm{~m}^{-3} \mathrm{~K}^{-1}\right]$ & 2.54 & 2.70 & 2.48 & 2.38 \\
$a\left[10^{-6} \mathrm{~m}^{2} \mathrm{~s}^{-1}\right]$ & 0.35 & 0.32 & 0.35 & 0.37 \\
\hline
\end{tabular}

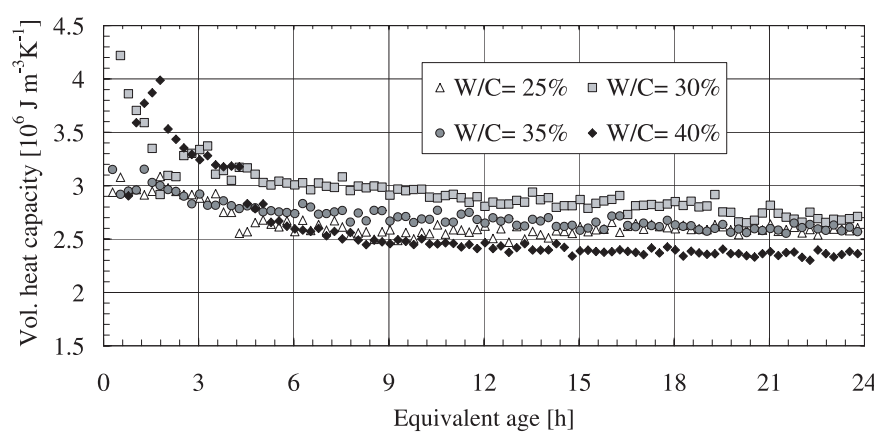

Fig. 15. Volumetric heat capacity of cement pastes with different $W / C$ ratios (test temperature: $294 \pm 1 \mathrm{~K}$ ).

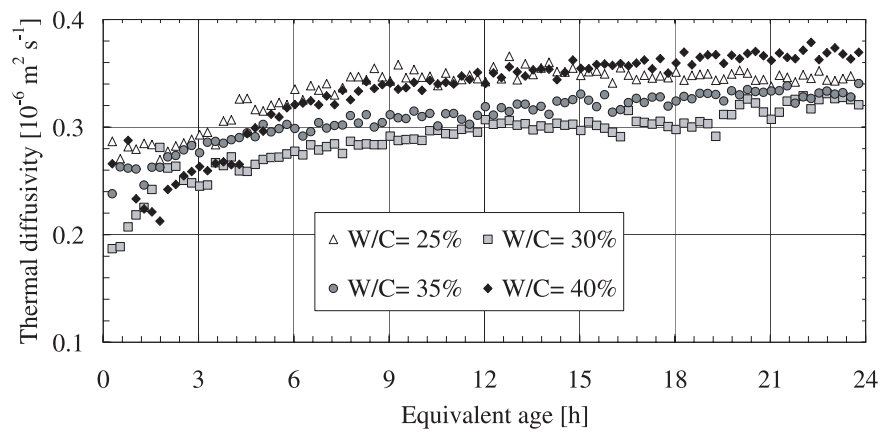

Fig. 16. Thermal diffusivity of cement pastes with different $W / C$ ratios (test temperature: $294 \pm 1 \mathrm{~K}$ ).

The evolution of the thermal diffusivity is plotted in Figure 16, for the studied cement pastes. The rise during the first hours of hydration can be explained by the increase of $\lambda$ (Fig. 14) and the diminution of $\rho C_{P}$ (Fig. 15).

The influence of the $W / C$ ratio on the evolution of thermal diffusivity, in the range of $W / C$ ratios investigated, is not very sensitive: the curves exhibit a similar evolution and reach, after 24 hours, similar thermal diffusivity values (see Tab. 4).

\subsection{HFIC method - Drying sand}

The sand sample is heated during 5 minutes through one of its larger faces $\left(4 \times 16 \mathrm{~cm}^{2}\right)$. The rise in temperature is measured by 6 thermocouples embedded at about $10 \mathrm{~mm}$ from the heating film (see Fig. 6). The precise thermocouple positions can be found in Table 5 .

Parabolic functions are chosen to represent the space evolution of $\lambda(x)$ and $\rho C_{P}(x)$. The computation time is about 1 hour.

Space evolutions of the water content and of $\lambda$ and $\rho C_{P}$ are presented in Figure 17.
Table 5. Positions of the thermocouples.

\begin{tabular}{ccccccc}
\hline Thermocouple & 1 & 2 & 3 & 4 & 5 & 6 \\
\hline$x[\mathrm{~mm}]$ & 11 & 19 & 30 & 50 & 79 & 118 \\
$z[\mathrm{~mm}]$ & 8.5 & 8.5 & 8 & 9 & 11 & 12 \\
\hline
\end{tabular}

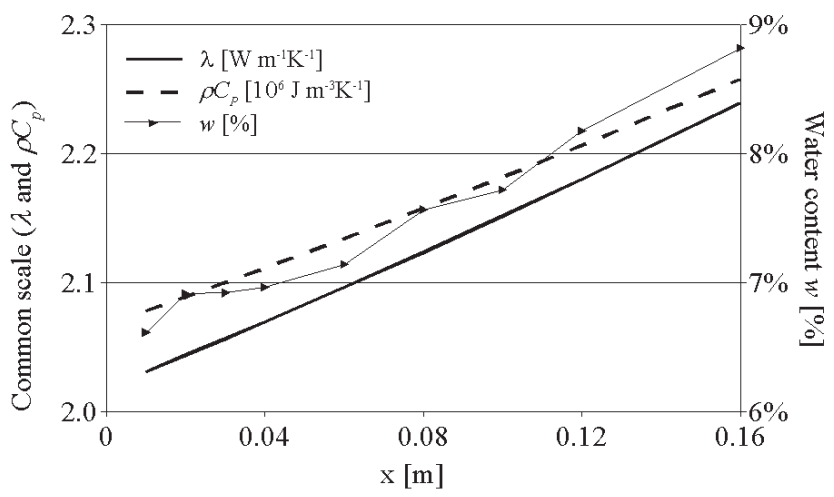

Fig. 17. Evolution of the thermal properties and water content of wet sand after 3 days of drying.

The water content evolves linearly from $6.5 \%$ to $8.8 \%$ between the sample free surface and the opposite face. A quasi linear evolution is also observed for both $\lambda$ and $\rho C_{P}$. From the drying face, their values increase and tend towards those found for wet sand (see Tab. 3).

These results show that drying is faster near the surface in contact with air. The replacement of liquid water by air/vapour mixture in the pores due to moisture exchange with the exterior provokes a diminution in the values of $\lambda$ and $\rho C_{P}$. Liquid water indeed exhibits higher thermal conductivity and volumetric heat capacity than air and vapour.

Figure 18 shows the evolution of the thermal properties versus the water content. The results are compared to those previously determined by Mounanga et al. [1] and Delacre [2]. Although differences can be observed concerning the heat capacity for a water content of $0 \%$, the values presented by Mounanga et al. [1] and Delacre [2] are in good agreement for both parameters. The difference at $w=0 \%$ can be related to the fact that the sand used by the authors is not of the same origin.

The results of the present paper are plotted on the same graph (see Fig. 18). The thermal conductivity values measured are consistent with those presented by Delacre [2]. The heat capacity values are situated slightly above those obtained by Delacre [2]. This can be, once again, attributed to the difference in composition of the two granular media. 


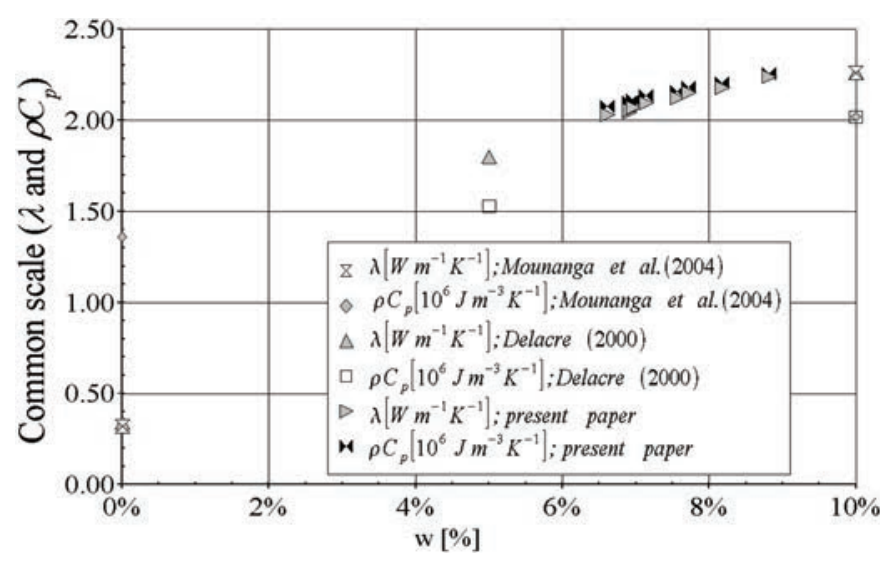

Fig. 18. Sand thermal properties vs. water content; comparison with previous studies.

\section{Conclusions}

In this paper, the "heated and non-heated wire" method (HnHW) and the "heating film with inverse calculation" method (HFIC) have been validated and applied to the determination of the thermophysical properties of evolutive materials. The results of these techniques have been compared with those obtained with the classical "heating film" method on a clayish material (HnHW method) and on hardened cement mortars and glass (HFIC method). A good agreement has been obtained between the different methods.

The HnHW method has then been used to study the thermal property evolution of hardening cement pastes as a function of their equivalent age. From tests performed at $294 \pm 1 \mathrm{~K}$, it is observed that, during the first day of hydration, the thermal conductivity increases slightly, the $W / C$ ratio having little influence on this parameter. The evolution of the heat capacity shows a strong initial diminution, explained by the gradual decrease in the amount of interstitial "free" water during hydration. Indeed, water has a higher heat capacity than the solid phase of the material. These tests have been carried out under uniform temperature conditions. However, cement hydration is a thermo-activated process. It would thus be of interest to study the influence of the temperature on $\lambda$ and $\rho C_{P}$ in order to propose values adapted to "realistic" conditions (e.g. large concrete structures with non uniform temperature field). The present study focusing on the influence of $W / C$ ratio, is a part of a larger research work. This latter includes the quantification of the temperature effect on the thermal properties [25].

The HFIC method has been applied to the study of drying sand. This technique together with the water content $(w)$ measurement permitted the determination of the evolution of the thermal properties versus $w$. The results, showing the decreasing of both thermal conductivity and volumetric heat capacity with the diminution of $w$, are in agreement with previous papers.

Further works will aim at studying the thermal properties of more complex evolutive materials. In the field of Civil Engineering, it is the case for hardening mortars and cement pastes submitted to drying, for which the HFIC method is particularly suitable.

The authors are grateful to Mrs. Véronique Baroghel-Bouny, head of the section "Microstructure and Durability of Concrete" at LCPC (Paris) as well as the technical staff of the section "Characterization and Study of Materials" for their assistance as regards the characterization of the cement used.

\section{Appendix A: Image method}

The HnHW method lays on theoretical developments explained hereafter. In this part, the equations are related to the temperature field around a continuous line source in an infinite medium. The resolution of the transient heat transfer equation in cylindrical coordinates yields the following relation:

$$
T(r, t)=\frac{P_{l}}{4 \pi \lambda} E_{1}\left(\frac{r^{2}}{4 a t}\right)
$$

where $r$ is the distance to the source $[\mathrm{m}], P_{l}$ the linear power $[\mathrm{W}], \lambda$ the thermal conductivity of the medium $\left[\mathrm{W} \mathrm{m}{ }^{-1} \mathrm{~K}^{-1}\right], a$ the thermal diffusivity $\left[\mathrm{m}^{2} \mathrm{~s}^{-1}\right]$ and $t$ the time $[\mathrm{s}] . E_{1}(x)$ is the exponential integral of $x$, that is to say:

$$
E_{1}(x)=\int_{x}^{+\infty} \frac{e^{-v}}{v} d v
$$

Let us consider a finite medium at the boundaries of which homogeneous conditions of the first kind are imposed (Fig. 5a). The time evolution of the temperature field can be found using the "image method". The problem results in the superimposition of the temperature fields generated by heat sources and sinks disposed according to a precise pattern. This pattern is given in Figure 5b. The positions of the sources and sinks are given hereafter (the origin is located on the heated wire and $n \in]-\infty ;+\infty[)$.

Position of the sources: $y=2 n h$

Position of the sinks: $y=(2 n+1) h$.

The following relation gives the temperature field in any point of the medium during the heating phase $T_{h}(r, t)$ :

$$
\begin{array}{r}
T_{h}(r, t)=\frac{P_{l}}{4 \pi \lambda} \sum_{n=-\infty}^{n=+\infty}\left\{E_{1}\left[\frac{x^{2}+(y-2 n h)^{2}}{4 a t}\right]\right. \\
\left.-E_{1}\left[\frac{x^{2}+(y-(2 n+1) h)^{2}}{4 a t}\right]\right\}
\end{array}
$$

It can also be used to calculate the temperature field after heating $T_{c}(r, t)$. In this case, heat sinks are superimposed to the heat sources, and inversely, which yields the 


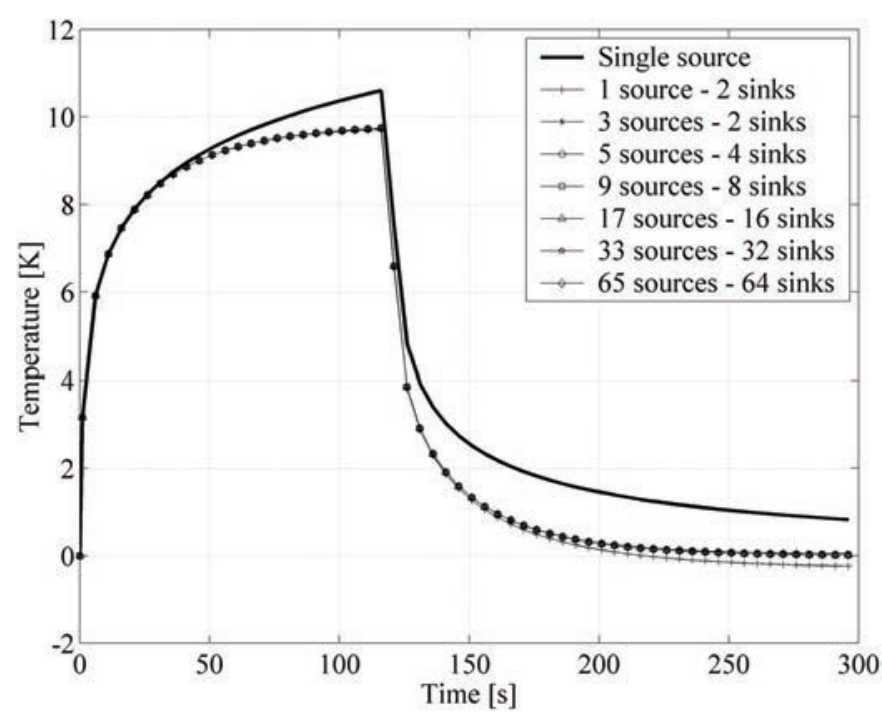

Fig. 19. Temperature time evolution of the heated wire; influence of the number of sources and sinks.

following relation:

$$
\begin{aligned}
T_{c}(r, t) & =\frac{P_{l}}{4 \pi \lambda} \sum_{n=-\infty}^{n=+\infty} E_{1}\left[\frac{x^{2}+(y-2 n h)^{2}}{4 a t}\right] \\
& -E_{1}\left[\frac{x^{2}+(y-(2 n+1) h)^{2}}{4 a t}\right] \\
& -\frac{P_{l}}{4 \pi \lambda} \sum_{n=-\infty}^{n=+\infty} E_{1}\left[\frac{x^{2}+(y-2 n h)^{2}}{4 a\left(t-t_{h}\right)}\right] \\
& -E_{1}\left[\frac{x^{2}+(y-(2 n+1) h)^{2}}{4 a\left(t-t_{h}\right)}\right]
\end{aligned}
$$

Relations A.3 and A.4 are rigorous, but converge rapidly. In order to check for the convergence of these series, tests are carried out. The varying parameter is the number of sources and sinks whose temperature field is taken into account. The profile for a single source is given as a reminder. In these simulations, the medium width is $20 \mathrm{~mm}$, the linear power is $20 \mathrm{~W} \mathrm{~m}^{-1}$, the distance between the wires is $15 \mathrm{~mm}, \lambda$ is $1 \mathrm{~W} \mathrm{~m}^{-1} \mathrm{~K}^{-1}, a$ is equal to $10^{-6} \mathrm{~m}^{2} \mathrm{~s}^{-1}$ and $t_{h}$ is $120 \mathrm{~s}$.

For the heated wire, Figure 19 shows that the number of sinks and sources has little influence on the temperature. The differences appearing at the end of the observed post-heating phase remain low. This shows the rapid convergence of the series given by equation A.4, near the source.

For the non-heated wire, no difference is observed during the heating phase (see Fig. 20). Only the post-heating phase exhibits a discrepancy between the profiles calculated with only few sources and sinks.

To calculate the temperature evolution of the heated and non-heated wires, it is possible to use a little number of sources and sinks to know the heating phase. For the post-heating phase, it is necessary to increase the number of reflections of the original source to get a better accuracy.

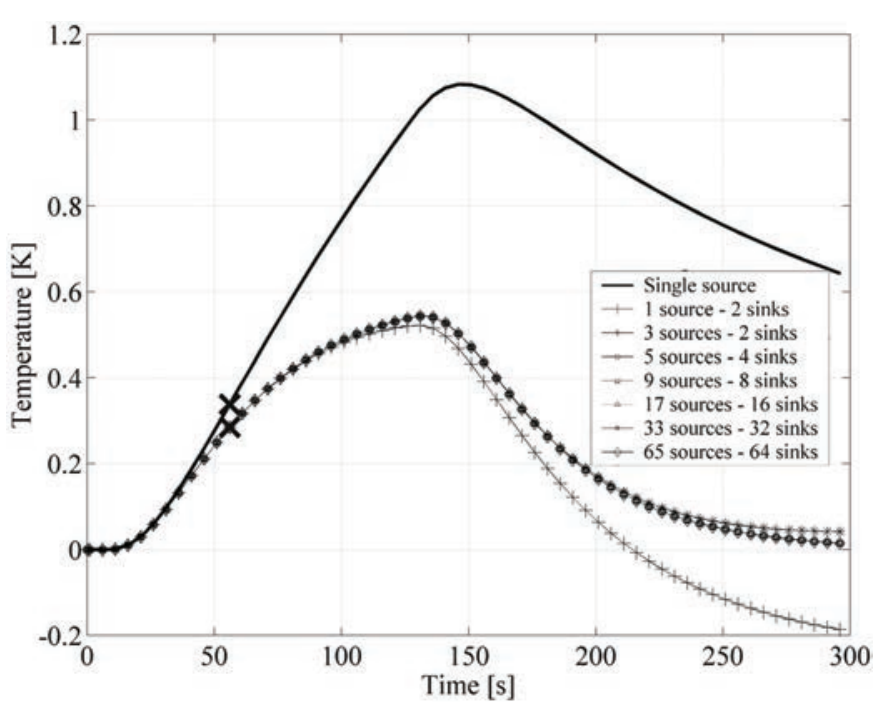

Fig. 20. Temperature time evolution of the non-heated wire; influence of the number of sources and sinks (large cross: point of inflection).

For the determination of $\rho C_{P}$ from equation (11) as explained in Section 3.1.2, only two sinks are used to correct the experimental data since the point of inflection is located in the heating phase during which the number of heat sources and sinks has little influence on the material temperature evolution.

\section{Appendix B: Discretization of the problem}

The heat conduction equation together with the boundary conditions (heat flux is null on the medium boundaries excepted on the lower face receiving the heat flux $\Phi$, Fig. 21) and initial condition (temperature is null at time $t=0$ ) can be used to determine the temperature field in the medium. In some cases, the thermophysical properties can evolve not only through space but also over time as the material internal structure changes. If this change is slow enough as compared with the experiment duration, the time evolution can be neglected. The system to be solved can thus be written as follow:

$$
\begin{gathered}
\rho C_{P}(x) \frac{\partial T}{\partial t}=-\vec{\nabla} \cdot(-\lambda(x) \vec{\nabla} T) \\
\rho C_{P}(x) \frac{\partial T}{\partial t}=\lambda(x)\left(\frac{\partial^{2} T}{\partial x^{2}}+\frac{\partial^{2} T}{\partial z^{2}}\right)+\frac{\partial \lambda}{\partial x} \frac{\partial T}{\partial x} \\
\left\{\begin{array}{l}
-\left.\lambda(x=0) \frac{\partial T}{\partial x}\right|_{x=0 ; z}=0 \\
-\left.\lambda\left(x=L_{x}\right) \frac{\partial T}{\partial x}\right|_{x=L_{x} ; z}=0 \\
-\left.\lambda(x) \frac{\partial T}{\partial z}\right|_{x ; z=0}=\Phi \\
-\left.\lambda(x) \frac{\partial T}{\partial z}\right|_{x, z=L_{z}}=0 \\
T(x ; z ; t=0)=0 .
\end{array}\right.
\end{gathered}
$$

Functions $\lambda(x)$ and $\rho C_{P}(x)$ describe the spatial variations of the thermal conductivity and of the volumetric specific 


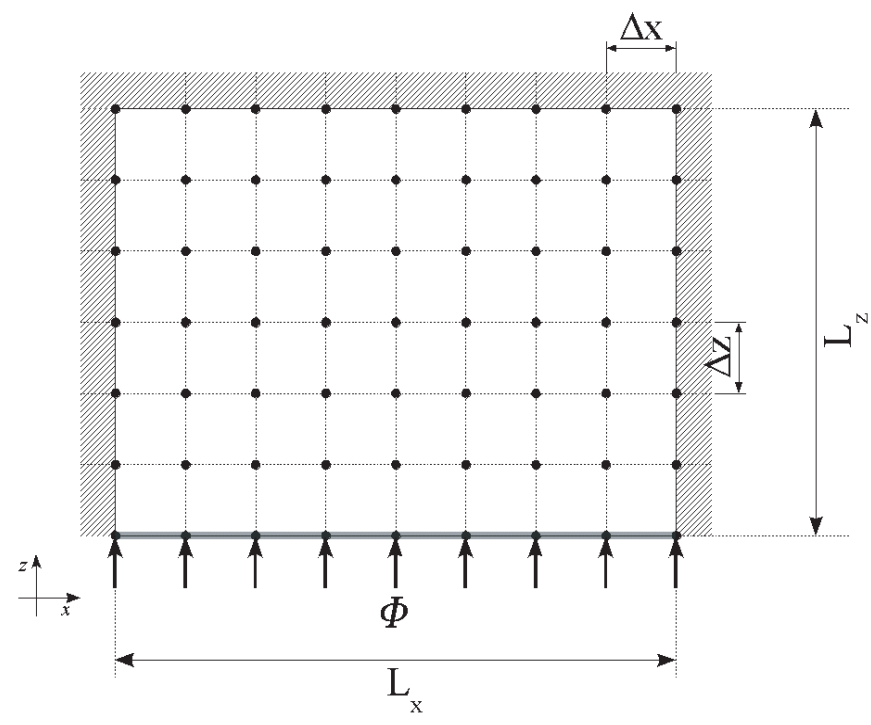

Fig. 21. Scheme of the problem.

heat respectively. The problem is thus non linear and must be solved numerically.

The discretization can be done by the finite difference method. Özışık [17] explains the different techniques that can be used to make the discretization. Central differences and the ADI method (Alternating Direction Implicit) are chosen to approximate the heat conduction equation. The ADI method consists in expressing the spatial derivatives with an implicit scheme in one direction and an explicit scheme in the other one. The directions of implicitness and explicitness are inverted at each time step. The advantage of this method is to obtain a linear system of equations that is unconditionally stable.

The following approximations are employed to express the spatial and temporal derivatives of the temperature field:

$$
\left\{\begin{array}{l}
\frac{\partial T}{\partial x}=\frac{T_{i+1, j}^{n}-T_{i-1, j}^{n}}{2 \Delta x} \\
\frac{\partial T}{\partial z}=\frac{T_{i, j+1}^{n}-T_{i, j-1}^{n}}{2 \Delta z} \\
\frac{\partial^{2} T}{\partial x^{2}}=\frac{T_{i+1, j}^{n}-2 T_{i, j}^{n}+T_{i-1, j}^{n}}{\Delta x^{2}} \\
\frac{\partial^{2} T}{\partial z^{2}}=\frac{T_{i, j+1}^{n}-2 T_{i, j}^{n}+T_{i, j-1}^{n}}{\Delta z^{2}} \\
\frac{\partial T}{\partial t}=\frac{T_{i, j}^{n+1}-T_{i, j}^{n}}{\Delta t}
\end{array}\right.
$$

$T_{i, j}^{n}$ is the temperature at time step $n$ and at the node located by $i$ along $x$ and $j$ along $z . \Delta x$ and $\Delta z$ are the space step. In the following calculi, $\Delta x=\Delta z$. $\Delta t$ is the time step.

If the implicitness direction is $x$ and the explicitness direction is $z$, the heat conduction equation can be approximated as (general term):

$$
\begin{gathered}
{\left[\left(b_{i}-a_{i}\right) T_{i-1, j}+\left(1+2 a_{i}\right) T_{i, j}-\left(a_{i}+b_{i}\right) T_{i+1, j}\right]^{n+1}} \\
=\left[a_{i} T_{i, j-1}+\left(1-2 a_{i}\right) T_{i, j}+a_{i} T_{i, j+1}\right]^{n}
\end{gathered}
$$

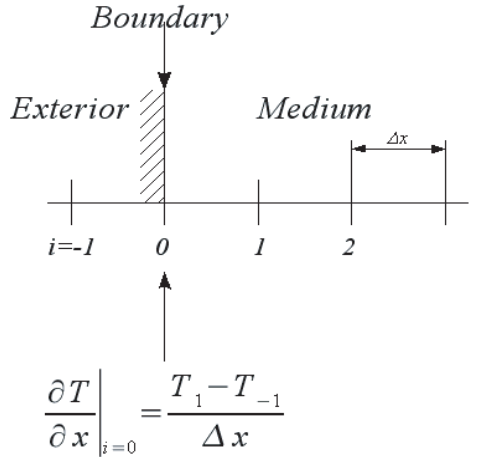

Fig. 22. Method of the fictitious node.

where parameters $a_{j}$ and $b_{j}$ are given by the following expressions:

$$
a_{i}=\frac{\lambda_{i} \Delta t}{\left(\rho C_{P}\right)_{i} \Delta x^{2}} ; \quad b_{i}=\left.\frac{\partial \lambda}{\partial x}\right|_{i} \frac{1}{2\left(\rho C_{P}\right)_{i}} \frac{\Delta t}{\Delta x}
$$

Inverting the directions of explicitness and implicitness yields the following general term:

$$
\begin{aligned}
& {\left[-a_{i} T_{i, j-1}+\left(1+2 a_{i}\right) T_{i, j}-a_{i} T_{i, j+1}\right]^{n+1}=} \\
& \quad\left[\left(a_{i}-b_{i}\right) T_{i-1, j}+\left(1-2 a_{i}\right) T_{i, j}+\left(a_{i}+b_{i}\right) T_{i+1, j}\right]^{n}
\end{aligned}
$$

Equations (B.5, B.6) are the general terms given by the discretization of equation (B.2) inside the medium. To calculate the temperature field, it is necessary to discretize the boundary conditions. These latter are treated through the method of the fictitious node. This allows to use central differences to express the spatial derivatives on the very boundaries. Figure 22 shows the principle of this method.

For example, if the heat flux at the node $i=0$ is null:

$$
-\lambda \frac{\partial T}{\partial x}=0 \Leftrightarrow T_{-1}=T_{1}
$$

In the expression of the general term at the node $i=$ $0, T_{-1}$ can be thus replaced by $T_{1}$ and equation (B.6) becomes:

$$
\begin{array}{r}
{\left[-a_{0} T_{0, j-1}+\left(1+2 a_{0}\right) T_{0, j}-a_{0} T_{0, j+1}\right]^{n+1}=} \\
{\left[\left(1-2 a_{0}\right) T_{0, j}+2 a_{0} T_{1, j}\right]^{n}}
\end{array}
$$

In the same way, the heat flux on the lower face of the medium is $\Phi$, that is to say:

$$
-\lambda \frac{\partial T}{\partial z}=\Phi \Leftrightarrow T_{i,-1}^{n}=T_{i, 1}^{n}-2 \frac{\Phi}{\lambda} \Delta x
$$

Replacing $T_{i,-1}$ by this latter expression in equation (B.5) yields:

$$
\begin{aligned}
& {\left[-a_{0} T_{i-1,0}+\left(1+2 a_{0}\right) T_{i, 0}-a_{0} T_{i+1,0}\right]^{n+1}=} \\
& \quad\left[\left(1-2 a_{0}\right) T_{i, 0}+2 a_{0} T_{i, 1}\right]^{n}+\left(a_{0}-b_{0}\right) R_{0}
\end{aligned}
$$


where:

$$
R_{i}=\frac{2 \Phi \Delta x}{\lambda_{i}}
$$

This has to be done for each face and for the explicit and implicit discretizations, 16 different equations describing the flux boundary conditions can be written: 2 equations for each face and 2 equations for each corner. These latter equations are obtained by taking into account the flux on each face determining the corner. They can be found by combining the equations written for each face. For example, for a corner located at the junction of an insulated face and a face receiving the flux $\Phi(i=0$ and $j=0)$, equation (B.5) becomes:

$$
\begin{aligned}
& {\left[\left(1+2 a_{0}\right) T_{0,0}-2 a_{0} T_{1,0}\right]^{n+1}} \\
& \quad=\left[\left(1-2 a_{0}\right) T_{0,0}+2 a_{0} T_{0,1}\right]^{n}+\left(a_{0}-b_{0}\right) R_{0}
\end{aligned}
$$

The discretization yields a linear system of equations in the shape of two tridiagonal matrices for odd time steps and two others for even time steps:

$-A_{z} T^{2 n}=B_{x} T^{2 n-1}+C_{x}$ implicit in $z$ and explicit in $x$ - $A_{x} T^{2 n+1}=B_{z} T^{2 n}+C_{z}$ explicit in $z$ and implicit in $x$

$A_{z}, A_{x}, B_{z}, B_{x}$ are tridiagonal matrices and $C_{x}$ and $C_{z}$ two vectors containing the flux boundary conditions.

The resolution of this system requires the inversion of matrices $A_{z}$ and $A_{x}$ :

$$
\begin{aligned}
& T^{2 n}=A_{z}^{-1}\left(B_{x} T^{2 n-1}+C_{x}\right) \\
& T^{2 n+1}=A_{x}^{-1}\left(B_{z} T^{2 n}+C_{z}\right) .
\end{aligned}
$$

The initial temperature is null at each node of the medium:

$$
T^{0}=\left[\begin{array}{c}
0 \\
\vdots \\
0
\end{array}\right] \text {. }
$$

\section{References}

1. P. Mounanga, G. Bastian, G. Bron, R. Coué, Eur. Phys. J. Appl. Phys. 26, 65 (2004)

2. E. Delacre, Ph.D. thesis, University of Artois, 2000
3. G. Bastian, Rev. Phys. Appl. 22, 431 (1987)

4. P. Acker, J.-F. Torrenti, F. Ulm, Comportement du béton au jeune âge (Hermès Science Publications, Paris, 2004)

5. J.P. Bournazel, Ph.D. thesis, University of Paris VI, 1992

6. O. Coussy, T. Lassabatère, in Congrès Français de Thermique, SFT 2004, Presqu'̂̀le de Giens (2004)

7. P. Crausse, J.-P. Laurent, B. Perrin, Rev. Gén. Therm. 35, 95 (1996)

8. G. Bastian, Ph.D. thesis, ENSM and University of Nantes, 1989

9. Ch. Moyne, Ph.D. thesis, Institut National Polytechnique de Lorraine, 1987

10. J.R. Philip, D.A. De Vries, T. Am. Geophys. Union 38, $222(1957)$

11. D.R. Flynn, National Institute of Standards and Technology, Final report GCR 99-767, 1999

12. G. Bastian, A. Khelidj, Bull. Liaison Labo. Ponts et Chaussées 200, 25 (1995)

13. G. Bastian, Rev. Phys. Appl. 17, 217 (1982)

14. J. Martinet, Éléments de Thermocinétique : Conduction de la Chaleur (Technique et Documentation-Lavoisier, Paris, 1989)

15. M. Abramowitz, I.A. Stegun, Handbook of Mathematical Functions (Dover Pub. Inc., New York, 1972)

16. H.S. Carslaw, J.C. Jaeger, Conduction of Heat in Solids, 2nd edn. (Clarendon Press, Oxford, 1959)

17. N. Özışı, Heat Conduction, 2nd edn. (Wiley-Interscience Publication, New York, 1993)

18. W.H. Press, B.P. Flannery, S.A. Teukolsky, W.T. Vetterling, Numerical Recipes: The Art of Scientific Computing (Cambridge University Press, New York, 1986)

19. G. Bastian, Rev. Phys. Appl. 24, 1057 (1989)

20. G. de Schutter, L. Taerwe, Mag. Con. Res. 47, 203 (1995)

21. G.J. Gibbon, Y. Ballim, Mag. Con. Res. 50, 229 (1998)

22. N.J. Carino, H.S. Lew, in Proceedings of the 2001 Structures Congress \& Exposition, Washington, DC, 2001, edited by P.C. Chang (American Society of Civil Engineers, Reston, Virginia, 2001), p. 1

23. P. Mounanga, A. Loukili, A. Khelidj, in Proceedings of the Seventh CANMET/ACI International Conference, Berlin, 2003, edited by V.M. Malhotra (ACI, Farmington Hills, 2003), p. 513

24. D.P. Bentz, V. Waller, F. de Larrard, Cem. Con. Res. 28, 285 (1998)

25. P. Mounanga, Ph.D. thesis, University of Nantes, 2003 